\title{
Les Teneurs Métalliques Et Paramètres Physico- Chimiques De L'eau Et Du Sédiment De Oued Beht, Au Niveau De Sidi Kacem Et De Oued R'dom Au Niveau De Sidi Slimane
}

\author{
Qaouiyid Aziz \\ Hmima Hajar \\ Houri Kaoutar \\ Saad Ilham \\ Saad EI Hassania \\ Mohamed Fadli
}

Laboratoire Santé, Nutrition et Environnement, Faculté des Sciences Kenitra, Université Ibn Tofail, Maroc

doi: 10.19044/esj.2016.v12n24p186 URL:http://dx.doi.org/10.19044/esj.2016.v12n24p186

\section{Abstract}

In the present study we evaluated the physicochemical characteristics and the degree of the metallic contamination, in spring and summer, of the areas exposed to discharges of urban and industrial waste water: Sidi Slimane which rejects its waste in Oued Beht, and Sidi Kacem, which rejects its waste on Oued R'dom. The two water courses are tributaries of Oued Sebou, the most polluted river in Morocco. For physico-chemistry, the results showed that for both areas exposed to waste, the $\mathrm{pH}$ is alkaline, the temperature is between $20^{\circ} \mathrm{C}$ to $26^{\circ} \mathrm{C}$ depending on the season, the average conductivity is very high, dissolved oxygen levels are low especially in summer, BOD5 and BCO show that the fraction of non-biodegradable waste is no negligible, the water hardness is not very high but it is more calcium than magnesium, an average level of alkalinity, an average level of orthophosphate, sulfates and nitrogenous substances. Similarly, the results showed that in areas exposed to discharges of the two cities, the metal contamination of the sediment is higher than that of water contamination although this remains nearly an average degree of contamination in these two compartments of the biotope. Furthermore, in the study areas, Oued R'dom showed a slightly higher assimilative capacity than Oued Beht except for Aluminium and Chrome. Also note that, for the same area of study, the factor "Season" influences the physico-chemical and the values of metallic chemical elements of the water and sediment. 
Keywords: Physico-chemical characteristics, Metal Pollution, Water, Sediment, Oued Beht, R'dom Oued, Morocco

\section{Résumé}

Dans le présent travail nous avons évalué les caractéristiques physico-chimiques et le degré de contamination printanier et estival des zones exposées aux rejets des eaux usées urbaines et industrielles de la ville de Sidi Slimane, rejetées sur Oued Beht, et de Sidi Kacem, rejetées sur Oued R'dom. Les deux cours récepteurs de ces rejets sont des affluents de Oued Sebou, rivière la plus polluée du Maroc. Pour les analyses physicochimiques, les résultats ont montré que, pour les deux zones exposées aux rejets, le $\mathrm{pH}$ est alcalin, la température est entre $20^{\circ} \mathrm{C}$ à $26^{\circ} \mathrm{C}$ selon la saison, la conductivité est moyenne, le taux d'oxygène dissous est faible surtout en été, des DBO5 et BCO qui montre que la fraction des rejets non biodégradables n'est négligeable, une dureté des eaux moyenne mais qui est plus calcique que magnésique, une alcalinité moyenne, des teneurs moyennes en orthophosphates, sulfates et substances azotées. De même, les résultats ont montré que, dans les zones exposées aux rejets des deux villes, la contamination métallique du sédiment est plus élevée que celle de l'eau quoique cette contamination reste moyenne pour ces deux compartiments du biotope. En outre, dans les zones étudiées, Oued R'dom a montré une capacité d'autoépuration légèrement plus élevée que celle de Oued Beht sauf pour l'Aluminium et le Chrome. A noter également que, pour une même zone d'étude, le facteur "Saison » influence les valeurs physico-chimiques et métalliques de l'eau et du sédiment.

Mots-clefs : Caractéristiques physico-chimique, Pollution métallique, Eau, Sédiment, Oued Beht, Oued R’dom, Maroc

\section{Introduction}

L’appréciation de la qualité des eaux de surface peut s'effectuer par la mesure de paramètres physico-chimiques et la teneur des éléments métalliques ainsi que par la détermination de la qualité et la fréquence d'organismes et de micro-organismes aquatiques considérés comme indicateurs de la qualité de l'eau (Bli-Effert et Perraud 2001). En effet, les métaux lourds par exemple sont biopersistants et certains sont même cancérigènes pour l'homme, perturbent les écosystèmes, détériorent les sols, les eaux de surface, les forêts et bio s'accumulent le long des chaînes trophiques. Ainsi, certains cours d'eau exposés à des facteurs écologiquement nocifs sont exposés à une dégradation perdent une partie de leurs services environnementaux (Walmsley, 2002). Ainsi, une 
eutrophisation des eaux de ces cours peut entrainer une modification de la dynamique de la biocénose du milieu (Aguilar-Ibarra, 2004 ; Chikou, 2006) notamment.

Par ailleurs, dans nombreux pays, sous l'influence de l'essor démographique et la multiplication accélérée des activités industrielles, agricoles et touristiques, le besoin en eau ne cesse d'augmenter. En outre, la dégradation de la qualité des eaux de nombreux hydrosystème aggrave la réduction de la disponibilité de cette substance en eau ; le Maroc en est un cas. La surveillance de la qualité des eaux des cours et accumulations d'eau est donc d'un intérêt capital pour la préservation cette substance vitale.

Dans ce cadre, l'étude de la qualité des eaux de Oued Beht et Oued R'dom, deux cours d'eau du nord-est du Maroc, revêt un intérêt particulier du fait que leurs bassins versant subissent un stresse écologique important d'origine anthropique notamment une agriculture intensive et des rejets dans le milieu d'effluents domestiques et industrielles sans traitement efficace préalable. Ainsi, comme l'a indiqué Leclerq (2001), pour un hydrosystème donné, la détermination des caractéristiques physico-chimiques, et la détermination des teneurs des éléments métalliques permet d’évaluer sa qualité et, par suite, sa productivité. Plus précisément, le présent travail contribue à l'étude spatio-temporelle des paramètres physico-chimiques et métalliques de oued Beht et Oued R'dom en amont, au niveau, et en aval de la ville Sidi Slimane pour Oued Beht, et en amont, au niveau, et en aval de la ville de Sidi Kacem pour Oued R'dom.

\section{Matériels at Méthodes}

\section{Milieu d'étude}

Le bassin de Sebou couvre deux principales plaines du Maroc, la plaine du Sais et la plaine du Gharb, connaît une pollution d'ordre organique et minérale causée par les décharges urbaines et industrielles des grandes agglomérations humaines implantées aux bordures des fleuves (Abdellaoui, 1990 ; Azzaoui, 1999)

Le bassin versant de Sebou est subdivisé en trois parties: haut Sebou, moyen Sebou et bas Sebou. Ce dernier est traversé par oued Beht et oued R'dom qui potentiellement sont chargés par des polluants qui proviennent des agglomérations et des installations industrielles situées au niveau de nombreuses villes telles que Meknès, Sidi Kacem, Khemisset, Sidi Slimane et Dar Gueddari (Fekhaoui et al., 1996). Oued R'dom, reçoit les eaux usées urbaines et/ou industrielles de nombreuses agglomérations humaines dont les villes de Meknès et Sidi Kacem. Oued Beht traverse plusieurs villes et agglomérations urbaines tels que Sidi Slimane et Sidi Yahia. Le phénomène de pollution constitue un danger direct pour l'environnement aquatique et indirectement pour les êtres vivants végétaux 
et animaux de ces deux cours d'eau. Ainsi, le présent travail contribue à l'évaluation de la qualité physico-chimique et métallique par le biais de l'estimation des valeurs ou teneurs de 16 paramètres physico-chimiques et les teneurs de 8 éléments métalliques lourds en plus de celle du fer. Ces mesures ont été effectuées au printemps et en été de 2014 dans trois sites (BI, B2 et B3) situés au niveau de Oued Beht et trois sites situés au niveau de Oued R'dom (R1, R2 et R3) (Fig. 1). B1 est située en amont de Sidi Slimane, B2 au niveau de cette ville et B3 à $6 \mathrm{~km}$ en aval de B2. R1 est située en amont de Sidi Kacem, R2 au niveau de Sidi Kacem, R3 à 6 km en aval de cette ville.

\section{Echantillonnage}

Le prélèvement, le transport et la conservation des échantillons d'eau à analyser ont été faits suivant le protocole du laboratoire de contrôle de la qualité des eaux de l'ONEP indiqué dans la note technique " Prélèvement, conditionnement et stockage des échantillons d'eau " (Abouzaid) et Duchesne, 1984). Les prélèvements du sédiment ont effectués jusqu’à une profondeur de $4 \mathrm{~cm}$ de la surface. Dans chaque compagne le nombre de prélèvement par stations est trois.

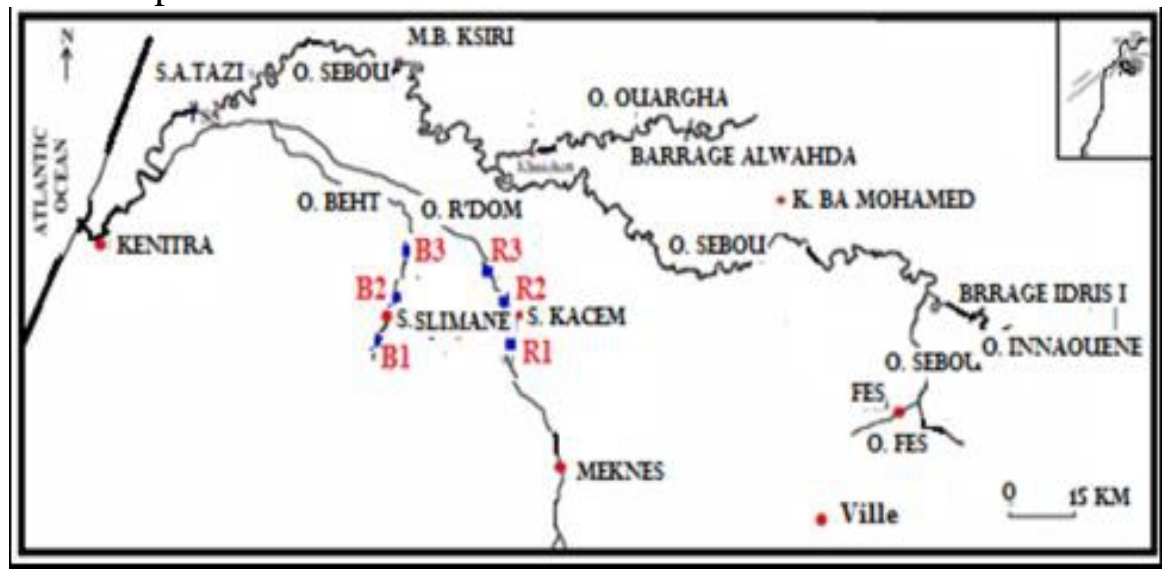

Figure 1 : Oueds et Stations étudiées

Par ailleurs, les méthodes de mesure des paramètres physicochimiques réalisées in situ (température, conductivité et oxygène dissous) ont été effectuées par appareillage électronique (conductimètre, salinomètre et oxymètre de type WTW, référence 320).

Pour les mesures effectuées au laboratoire, selon le paramètre physico-chimique à mesurer, la méthodologie et la norme de mesure suivies diffèrent : la DBO5 (Norme Afnor T90-103), la DCO (Norme Afnor T90101), les nitrates (DIN 38405-D9.2), les ions ammonium (Norme Afnor T90015, Les sulfates (Norme Afnor T90-040); pour les nitrites, les ortho- 
phosphates et les duretés totale (Rodier ; 1984) le chlorures l'alcalinité les méthodes suivies sont celles dont les principes sont décrits dans par Rodier (1984)

Les teneurs des éléments métalliques dans l'eau et dans le sédiment ont été effectués par ICP-AES qui est une technique basée sur le couplage d'une torche à plasma et d'un spectromètre d'émission atomique. Le degré de contamination métallique des stations prospectées a été effectué par calcul de l'Indice de contamination (Ic). A noter que pour un élément métallique donné, dans une station $\mathrm{x}$, l'indice de contamination du milieu par cet élément est :

$$
\text { Ic }=\frac{\text { Teneur de l'élément métallique sur la station } \mathrm{x}}{\text { Teneur du même élément sur la station de référence }}
$$

Pour un Ic proche de ' 1 ', on considère que le site n'est pas ou peu contaminé par les métaux. C’est au-delà de 2 que les auteurs (Rodier, 2009). admettent que la station est soumise à un début de contamination. De même, la contamination en termes d'importance de la valeur de Ic permet la distinction de quatre classes de contamination établies dans l'ordre de la classification suivante :

-La classe 1 : de 0 à 3 (contamination faible);

-La classe 2 : de 4 à 10 (contamination moyenne);

-La classe 3 : de 10 à 20 (contamination importante) ;

-La classe $4:>20$ (contamination excessive).

\section{Résultats et discussion}

Les tableaux I, II, III et IV groupent les valeurs des paramètres physico-chimiques et des éléments métalliques, et de l'indice de contamination métalliques des stations prospectées :

\section{Paramètres physico-chimiques}

Tableau I : Valeurs des variables physicochimiques dans trois stations (B1, B2 et B3) d'Oued Beht

\begin{tabular}{|c|c|c|c|c|c|c|c|c|c|}
\hline \multirow[b]{2}{*}{ Variables } & \multicolumn{3}{|c|}{ B1 } & \multicolumn{3}{|c|}{$\mathrm{B} 2$} & \multicolumn{3}{|c|}{ B3 } \\
\hline & 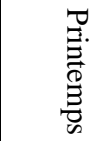 & $\stackrel{\mathbb{T}}{\vec{D}}$ & $\begin{array}{l}\Xi \\
\stackrel{\Xi}{0} \\
\stackrel{\Xi}{\Xi}\end{array}$ & 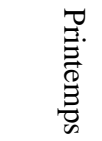 & $\underset{\vec{D}}{\vec{T}}$ & 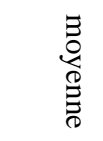 & 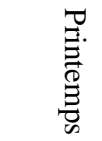 & $\stackrel{\vec{T}}{\vec{D}}$ & 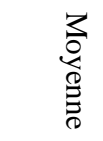 \\
\hline $\mathrm{Te}$ & 20,5 & 26,0 & 23,3 & 21,6 & 27 & 21,6 & 20,3 & 27,2 & 23,8 \\
\hline $\mathrm{pH}$ & 7,2 & 8,1 & 7,6 & 7,6 & 7,7 & 7,7 & 7,2 & 7,3 & 7,3 \\
\hline Cl- & 76,5 & 192,5 & 134,5 & 219,2 & 260,8 & 240,0 & 239,6 & 296,7 & 268,1 \\
\hline $\mathrm{CE}$ & 2453,0 & 3006,0 & 2729,5 & 3259,0 & 3981,0 & 3620,0 & 2833,0 & 3008,0 & 2920,5 \\
\hline Od & 5,2 & 6,6 & 5,9 & 2,0 & 3,1 & 2,6 & 4,0 & 3,1 & 3,6 \\
\hline DBO5 & 18,0 & 31,5 & 24,8 & 42,6 & 153,6 & 98,1 & 22,1 & 31,2 & 26,7 \\
\hline DCO & 26,6 & 87,7 & 57,2 & 53,0 & 180,0 & 116,5 & 45,0 & 91,0 & 68,0 \\
\hline Dureté totale & 132,1 & 212,1 & 172,1 & 158,8 & 238,8 & 198,8 & 157,7 & 221,2 & 189,5 \\
\hline
\end{tabular}




\begin{tabular}{|c|c|c|c|c|c|c|c|c|c|} 
Ca++ & 124,0 & 173,0 & 148,5 & 128,7 & 191,6 & 160,2 & 129,7 & 187,7 & 158,7 \\
\hline Mg++ & 8,1 & 29,1 & 18,6 & 30,1 & 47,2 & 38,7 & 28,0 & 33,5 & 30,8 \\
\hline $\begin{array}{c}\text { Alcalinité } \\
\text { (CaCO3) }\end{array}$ & 155,0 & 256,0 & 205,5 & 376,0 & 1771,0 & 1073,5 & 164,0 & 2014,0 & 1089,0 \\
\hline Orthophosphates & 0,0 & 0,0 & 0,0 & 0,0 & 0,1 & 0,0 & 0,0 & 0,0 & 0,0 \\
\hline Sulfates & 8,2 & 67,9 & 38,1 & 39,5 & 120,0 & 79,8 & 25,6 & 86,9 & 56,2 \\
\hline Nitrates & 0,1 & 2,3 & 1,2 & 0,2 & 3,2 & 1,7 & 0,2 & 2,2 & 1,2 \\
\hline Nitrites & 0,1 & 0,1 & 0,1 & 0,2 & 0,4 & 0,3 & 0,1 & 0,2 & 0,1 \\
\hline Ammo-nium & 3,6 & 3,7 & 3,6 & 4,3 & 7,5 & 5,9 & 3,2 & 6,4 & 4,8 \\
\hline
\end{tabular}

Tableau II : Valeurs des variables physicochimiques dans trois stations (R1, R2 et R3) de oued R'dom

\begin{tabular}{|c|c|c|c|c|c|c|c|c|c|}
\hline Variables & \multicolumn{3}{|c|}{ R1 } & \multicolumn{3}{|c|}{ R2 } & \multicolumn{3}{|c|}{ R3 } \\
\hline & $\begin{array}{l}\text { 总 } \\
\text { 营 } \\
\text { 总 }\end{array}$ & $\stackrel{\underline{D}}{\vec{D}}$ & 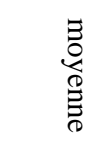 & 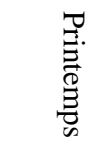 & 塄 & 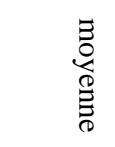 & $\begin{array}{l}\text { 哥. } \\
\text { 蛋 } \\
\text { 总 }\end{array}$ & $\stackrel{\text { 思 }}{\overrightarrow{0}}$ & 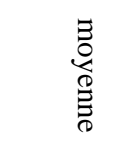 \\
\hline $\mathrm{Te}$ & 21,6 & 27,6 & 24,6 & 21,8 & 26,5 & 21,8 & 20,4 & 26,9 & 23,65 \\
\hline $\mathrm{pH}$ & 7,2 & 7,55 & 7,375 & 7,52 & 7,81 & 7,665 & 7,36 & 7,42 & 7,39 \\
\hline Cl- & 106,7 & 698,22 & 402,46 & 128,15 & $\overline{519,2}$ & 323,675 & 175,54 & 496,7 & 336,12 \\
\hline $\mathrm{CE}$ & 1480 & 1542 & 1511 & 1691 & 2034 & 1862,5 & 1787 & 1866 & 1826,5 \\
\hline Od & 5,4 & 9,25 & 7,325 & 2,1 & 2,9 & 2,5 & 3,7 & 2,9 & 3,3 \\
\hline DBO5 & 42,99 & 105,6 & 74,295 & 46,16 & 83,5 & 64,83 & 29,6 & 107,3 & 68,45 \\
\hline DCO & 110,3 & 122,8 & 116,55 & 163,7 & 131,3 & 147,5 & 56,3 & 19,18 & 37,74 \\
\hline Dureté totale & 135,5 & 178,5 & 157 & 156,2 & 215,91 & 186,055 & 165,91 & 228,23 & 197,07 \\
\hline $\mathrm{Ca}++$ & 120 & 160 & 140 & 138 & 176 & 157 & 128,11 & 188,1 & 158,105 \\
\hline $\mathrm{Mg}++$ & 15,5 & 18,5 & 17 & 18,2 & 29,91 & 24,055 & 27,8 & 40,22 & 34,01 \\
\hline $\begin{array}{c}\text { Alcalinité } \\
\text { (CaCO3) }\end{array}$ & 98 & 195 & 146,5 & 283 & 1063 & 673 & 122 & 1704 & 913 \\
\hline Orthophosphates & 0,02 & 0,025 & 0,0225 & 0,051 & 0,082 & 0,0665 & 0,034 & 0,041 & 0,0375 \\
\hline Sulfates & 6,12 & 58,36 & 32,24 & 48,57 & 9,22 & 28,895 & 6,86 & 27,44 & 17,15 \\
\hline Nitrates & 0,14 & 0,16 & 0,15 & 0,27 & 1,29 & 0,78 & 1,17 & 1,01 & 1,09 \\
\hline Nitrites & 0,12 & 0,1 & 0,11 & 0,14 & 0,17 & 0,155 & 0,14 & 0,15 & 0,145 \\
\hline Ammo-nium & 3,7 & 3,82 & 3,76 & 4,17 & 5,16 & 4,665 & 3,87 & 4,11 & 3,99 \\
\hline
\end{tabular}

\section{La température de l'eau}

Pour les deux cours d'eau prospectés, les résultats montrent que pour une même saison, la température ne varie pas significativement d'une station à l'autre. En effet, celle-ci tourne autour de $20^{\circ} \mathrm{C}$ au printemps et $26^{\circ} \mathrm{C}$ en été. Ainsi, ces valeurs notées ne dépassent pas les normes marocaines de 2002, et les températures des eaux des deux cours d'eau étudiés appartiennent à la classe moyenne à excellente.

A signaler que la température de l'eau, est un facteur écologique qui entraîne d'importantes répercutions écologiques (Leynaud, 1968). Elle agit sur la densité, la viscosité, la solubilité des gaz dans l'eau et la dissociation des sels dissous (Bertolini et Tani, 1995 ; Alenka, David, 1996 ; Baraud et al., 1999). Elle a un effet sur les réactions chimiques et biochimiques, et sur 
le développement et la croissance des organismes vivants dans l'eau et particulièrement les microorganismes (W.H.O, 1987).

\section{Le pH}

Le pH est alcalin dans les six stations des deux rivières et ne présente que de légères variations d'une station à l'autre et d'une saison à l'autre. Les valeurs plus alcalines en été et comme l’ont signalé (Ribolzi et al., 1993), cette alcalinité pourrait être liée aux terrains calcaires ou marno-calcaires jurassiques traversés par les cours d'eau.

A noter que le $\mathrm{pH}$ résume la stabilité de l'équilibre établi entre les différentes formes de l'acide carbonique qui interviennent dans le système tampon développé par les carbonates et les bicarbonates (Ezzaouaq, 1991 ; El Blidi et al., 2003 ; Himmi et al., 2003).

\section{Chlorures}

Les concentrations en ions chlorures relevées à Oued Beht varient de $76,45 \mathrm{mg} / \mathrm{l}$, notée au printemps en B1, et 296,7 mg/l, notée en été en B3. L'accumulationdes chlorures le long du cours d'eau et la diminution du débit de ce dernier explique cette augmentation par cumule des valeurs des chlorures. A Oued R'dom la valeur la plus faible $(106,7 \mathrm{mg} / \mathrm{l})$ est notée au printemps et la plus élevée $(698,22 \mathrm{mg} / \mathrm{l})$ en été en R1. Le faible débit de Oued Beht en été au niveau de R1 accentue le phénomène de l'évaporation des eaux du site. Toutes fois, pour les deux cours d'eau, les teneurs de chlorures notées ne dépassent pas les normes marocaines fixées à $750 \mathrm{mg} / \mathrm{l}$. Ainsi, l'eau est appartient a la classe «Bonne » des eaux superficielles dans les deux cours d'eau.

Par ailleurs, les chlorures sont des anions inorganiques importants contenus en concentrations variables dans les eaux naturelles, généralement sous forme de sels de sodium $(\mathrm{Nacl})$ et de potassium $(\mathrm{KCl})$. Ils sont souvent utilisés comme un indice de pollution (Nouayti et al., 2015) et ont une influence sur la faune et la flore aquatique ainsi que sur la croissance des végétaux (Lepengue et al., 1997).

\section{Conductivité Electrique}

Pour Oued Beht, les valeurs de la conductivité de l'eau sont entre $2453 \mu \mathrm{S} / \mathrm{cm}$ notée en automne en B1 et $3981 \mu \mathrm{S} / \mathrm{cm}$ enregistrée en été en B2. De même, les valeurs de Oued R'dom varient entre $1480 \mu \mathrm{S} / \mathrm{cm}$ enregistré au printemps en R1 et $2434 \mu \mathrm{S} / \mathrm{cm}$ enregistrée en été en R2. L'exposition des deux stations B2 et R2, qui sont exposées respectivement aux rejets des eaux usées de la ville de Sidi Slimane et ceux de Sidi Kacem, et la diminution des débits des cours d'eau en été pourraient être les causes principales. A noter 
que pour les deux cours d'eau, l'ensemble des valeurs notées restent inférieures à la norme marocaine des eaux de surface $(2700 \mu \mathrm{s} / \mathrm{cm})$.

Par ailleurs, la mesure de la conductivité constitue une bonne appréciation du degré de minéralisation d’une eau où chaque ion agit en fonction de sa concentration et sa conductivité. La conductivité électrique traduit le degré de minéralisation globale, et renseigne sur le taux de salinité. Elle a un rôle écologique important (Elhassan et al., 2012)

\section{Oxygène dissous}

Pour l'ensemble des stations prospectées, les teneurs les plus élevées ont été notées au printemps et celles les plus faibles en été. L’importance de la dégradation de la matière organique et l'augmentation de la température de l'été expliquent ce phénomène. De même, les teneurs d’oxygène dissous notées en B2 et en R2 sont plus faibles que celles notées en B1 ou R1, ou celles notées en B3 ou R3. Ceci peut être expliqué par la grande richesse des rejets urbains en matières organiques qui sont de grands consommateurs d’oxygène du milieu aquatique.

Par ailleurs, l’oxygène dissous dans l'eau est un élément fondamental qui intervient dans la majorité des processus biologiques (Mittal et Murad 1979 ; Deby et Deby-Dupont 1993 ; Lehninger et al., 1993 ; Droge 2002). Végétaux et animaux l'utilisent pour la respiration. L'oxygène participe également aux dégradations biochimiques et chimiques (Mittal et Murad 1979 ; Deby et Deby-Dupont 1993 ; Lehninger et al., 1993 ; Droge, 2002). Ainsi, une chute de l'oxygène dissous dans le milieu récepteur, phénomène qui conduira à l'asphyxie des espèces présentes.

\section{DBO5}

A oued Beht les valeurs oscille entre $18 \mathrm{mg} / \mathrm{l}$ notée au printemps en B1 et 153,56 mg/l notée en été en B2. A Oued R'dom, la valeur la plus petite (29,6 mg/l) a été notée au printemps en B3 et la valeur la plus élevée (107,3 $\mathrm{mg} / \mathrm{l}$ ) a été enregistrée au printemps en R3. L'évolution de la distribution des valeurs de la DBO5 notée au niveau de Beht ne suit pas donc la même allure que celle notée à Oued R'dom. Cette différence pourrait être due à la différence de la composition chimique et biologique des apports et rejets déversés par leur bassin versant notamment ceux des agglomérations urbaines riveraines.

Par ailleurs, en général, la demande biologique en oxygène sert de paramètre global pour l'évaluation de la pollution des eaux usées et constitue un bon indicateur de la teneur en matières organiques biodégradables d'une eau au cours des procédés d'autoépuration (Boutayeb et al., 2012). Ainsi, pour un hydrosystème donné, l’évaluation de la DBO permet de mesurer la qualité biologique de l'eau (Boutayeb et al., 2012). En effet, pour cet 
hydrosystème, l'eau analysée contient une quantité de matières organiques biodégradables, rejetées dans le milieu naturel, ces matières organiques vont être dégradées par voie biologique, ce qui va entrainer un développement de microorganismes aérobies. Ainsi, une valeur faible de la DBO5 indique une chute de l'oxygène dissous dans le milieu récepteur.

\section{$\mathrm{DCO}^{2}$}

Pour Oued Beht, la valeur la plus basse (26,5 mg/l) a été notée en automne en B1 et la valeur la plus élevée a été enregistrée en été en B2. Pour Oued R'dom la valeur la plus basse a été notée en été en R3 et la valeur la plus élevée a été enregistrée en été en R2. Ce sont les valeurs observées en R2 qui témoignent la richesse du milieu en usée brute.

Par ailleurs, la demande chimique en oxygène (DCO) permet d'évaluer la charge polluante des eaux usées. Ainsi, elle est particulièrement indiquée pour mesurer la pollution d'un effluent industrielle et pour contrôler les normes de rejet (Mercoiret, 2010 ; Boutayeb et al., 2012). L'eau potable ou les eaux des rivières doivent avoir une DBO5 proche de zéro jusqu'à quelques mg/l, les eaux usées d'origine domestique peuvent atteindre des valeurs proches de $300 \mathrm{mg} / \mathrm{l}$ tandis que les eaux usées provenant d'industries particulièrement polluantes (laiteries, distilleries) peuvent atteindre des valeurs de plusieurs dizaines de milliers de $\mathrm{mg} / \mathrm{l}$.

Par ailleurs, le rapport DCO/DBO5 donne une première estimation de la biodégradabilité de la matière organique d'un effluent donné (Mercoiret, 2010 ; Boutayeb et al., 2012.

\section{Dureté totale, Dureté Calcique (Ca++) et Dureté magnésique (Mg++)}

Pour Oued Beht, la dureté calcique varie de $124 \mathrm{mg} / \mathrm{l}$ à 187,71 mg/l. Moyennement, cette dureté augmente de l'amont en aval de la tranche des cours d'eau étudiés. En outre, dans la même station la dureté calcique et plus élevée en été. L'évaporation des eaux en été explique ce phénomène. Moyennement, comme à Oued Beht, la dureté calcique des eaux de Oued R'dom augmente d'amont en aval. A noter également, que avec des valeurs stationnelles moyennes plus élevées pour la dureté calcique, plus faibles pour la dureté magnésiques, et plus élevées pour la dureté totale l'évolution des valeurs de ces duretés suivent la même allure que celle de la dureté calcique.

A noter qu'au cours de son acheminement dans le sol, l'eau se charge naturellement en sels minéraux. Les teneurs en calcaire et magnésium dépendent de la nature des couches géologiques avec lesquelles l'eau s'est trouvée en contact. Une eau est dite "dure" ou "calcaire" lorsqu'elle est riche en sels de calcium et de magnésium ; c'est le cas des eaux ayant traversé des roches calcaires (Rodier, 2009). 


\section{Alcalinité (CaCO3-)}

A Oued Beht l'alcalinité la plus faible (155 mg/l) est enregistrée en automne en B1 et celle la plus élevée (2014 mg/l) en été en en B3. En outre, pour chaque station l'alcalinité estivale est plus élevée que celle printanières. Avec des valeurs proches, l'évolution de l'alcalinité à Oued R'dom à la même allure que celle notée à Oued Beht.

Par ailleurs, l'alcalinité est une mesure du pouvoir de l'eau à neutraliser les acides ; autrement dit, l’alcalinité mesure la résistance à tout changement de $\mathrm{pH}$. Ce pouvoir neutralisant de l'eau est attribué principalement à la présence de bicarbonates de calcium et de magnésium dissous dans l'eau. Ainsi, il y a un lien entre la dureté et l'alcalinité de l'eau (Kestemont, 2002).

\section{Orthophosphates et les sulfates}

Pour les deux cours d'eau les petites teneurs moyennes en orthophophates et en sulfates sont notées au printemps en B1 et R1 qui sont des stations situées en amont des rejets urbains ou industrielles et les valeurs moyennes les plus élevées sont notés en B2 ou R2, stations qui sont directement exposées à ces rejets. Ces rejets sont donc une importante source d'ortho-phosphate. De même, les phosphates sont utilisés dans l'agriculture comme engrais pour enrichir les fruits et légumes en source de phosphore (Anonyme, 2010). Ces engrais peuvent être d'origine organique (poudre d'os, arêtes de poissons, etc.) ou inorganique (attaque d'acide sur du minerai), ce qui est de plus en plus le cas, hormis en agriculture biologique où les engrais de synthèse sont interdits. Les algues utilisent ce phosphore minéral sous forme d'orthophosphates soit en l'absorbant directement soit en dégradant divers phosphates

organiques (Kestemont, 2002 ; Chahboun et al., 2014).

Par ailleurs, la plupart des composés sulfates proviennent de l'oxydation des minerais de sulfites, la présence de schistes, ou de déchets industriels. Les sulfates (SO4) peuvent être trouvés dans presque toutes les eaux naturelles. Ainsi, le sulfate est un des éléments majeurs des composés dissouts dans l'eau de pluie. Des concentrations importantes en sulfate dans l'eau peuvent avoir un effet laxatif important combiné avec le calcium et le magnésium, les deux composés majeurs de la dureté de l'eau (Zhou, 2010). Les normes de l'OMS suggérant un maximum de $250 \mathrm{mg} / \mathrm{l}$ en sulfates dans l'eau destinée à la consommation humaine. Au niveau des stations prospectées, les teneurs des sulfates sont inférieures à cette valeur.

\section{Nitrates, Nitrites et Ammonium}

L'évolution des teneurs des nitrates, des nitrites et de l'ammonium suit la même évolution des teneurs des orthophosphates et sulfates. Les 
petites teneurs moyennes sont notées au printemps en B1 et R1, qui sont des stations situées en amont des rejets urbains ou industrielles et les valeurs moyennes les plus élevées sont notés en B2 ou R2, stations qui sont directement exposées à ces rejets. Ces rejets sont donc une importante source de ces composés azotés. Notons que la présence de nitrates dans l'eau de consommation est principalement attribuable aux activités humaines (Santé Canada, 1992 ; Chartrand et al., 1999). En effet, l'utilisation de fertilisants synthétiques et de fumiers, associés aux cultures et à l'élevage intensifs, favorisent l'apparition de nitrates dans l'eau. Les installations septiques déficientes, de même que la décomposition de la matière végétale et animale, peuvent aussi être une source de nitrates dans l'eau (Levallois et Phaneuf, 1994 ; Gulis el al., 2001). Les nitrates (NO3-) et les nitrites (NO2-) sont des ions présents de façon naturelle dans l'environnement également. Ils résultent d'une nitrification de l'ion ammonium (NH4+), présent dans l'eau et le sol, qui est oxydé en nitrites par les bactéries du genre Nitrosomonas, puis en nitrates par les bactéries du genre Nitrobacter (Santé Canada, 1992 ; Chartrand et al., 1999). Les nitrates sont très solubles dans l'eau; ils migrent donc aisément dans la nappe phréatique lorsque les niveaux excèdent les besoins de la végétation (Santé Canada, 1992 ; Chartrand et al., 1999). La toxicité des nitrates résulte de leur réduction en nitrites et de la formation de méthémoglobine et de leur contribution possible à la synthèse endogène de composés N-nitrosés.

Les nitrates constituent le stade final de l'oxydation de l'azote, et représentent la forme d'azote au degré d'oxydation le plus élevé présent dans l'eau. Leurs concentrations dans les eaux naturelles sont comprises entre 1 et $10 \mathrm{mg} / \mathrm{l}$. Les teneurs en nitrates enregistrées au cours des deux compagnes de mesures varient entre 0,12 mg/l mesuré au printemps en B1 et 3,19 mg/l illustré en été en B2. Cependant, l'ensemble des teneurs de nitrates notées sont inférieures à $30 \mathrm{mg} / \mathrm{l}$, fixée par les normes marocaines. Il semble donc que, à l'état actuel, les eaux d'oued Beht et d'oued R'dom ne présentent pas des valeurs de nitrates menaçantes.

Pour l'ammonium, la teneur de cet élément traduit habituellement un processus de dégradation incomplet de la matière organique (Badraoui et al., 2000 ; Berdai el al., 2004). L'ammonium provient de la réaction de minéraux contenant du fer avec des nitrates. C'est donc un excellent indicateur de la pollution de l'eau par des rejets organiques d'origine agricole, domestique ou industriel (Badraoui et al., 2000 ; Berdai el al., 2004). Pour les teneurs de l'ammonium dans l'eau ayant des effet sur la santé et l'environnement, les normes de l'UE autorisent $0.5 \mathrm{mg} / \mathrm{l}$. Dépassant cette valeur il est peu toxique mais il peut provoquer plusieurs problèmes au niveau du milieu naturel (Zhao et al., 2008 ; Soaresa et al., 2011). Pour les plantes, une richesse du milieu en ammonium entraîne un déséquilibre dans leur 
alimentation et augmente leur fragilité vis-à-vis d'autres facteurs secondaires de stress. En outre, suivant les caractéristiques physico-chimiques des sols et des eaux cela peut déboucher, soit sur un enrichissement en azote, soit sur une acidification avec disparition de la faune et de la flore dans les cas extrêmes (Zhao et al., 2008 ; Soaresa et al., 2011).

\section{Teneurs et degrés de pollution métalliques}

\section{Teneurs métalliques dans l'eau et dans le sédiment}

La présence de ces métaux même à des faibles concentrations dans les eaux de surface peut avoir des impacts écologiques et sanitaires importants. Ainsi, nous avons évalué les teneurs de 8 métaux lourds et du fer. Les résultats sont illustrés dans les tableaux III et IV.

Tableau III : Teneurs des éléments métalliques dans trois stations B1, B2 et B3 de oued Beht

\begin{tabular}{|c|c|c|c|c|c|c|c|c|c|c|c|c|}
\hline Variables & & \multicolumn{3}{|c|}{ B1 } & \multicolumn{3}{|c|}{$\mathrm{B} 2$} & \multicolumn{3}{|c|}{ B3 } & \multirow[b]{2}{*}{$\stackrel{\overrightarrow{\mathfrak{f}}}{\mathrm{N}}$} & \multirow[b]{2}{*}{$\overline{\tilde{\omega}}$} \\
\hline & & 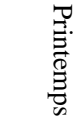 & 面 & 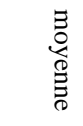 & $\begin{array}{l}\text { 兽 } \\
\text { 莺 }\end{array}$ & $\stackrel{\text { T) }}{\vec{D}}$ & 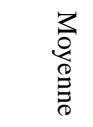 & 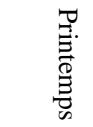 & $\stackrel{\text { T. }}{\vec{D}}$ & 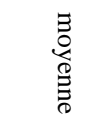 & & \\
\hline \multirow[b]{2}{*}{$\mathrm{Cd}$} & $\begin{array}{l}\text { M } \\
\stackrel{\Xi}{\Xi}\end{array}$ & 0,03 & 0,05 & 0,04 & 0,04 & 0,06 & 0,05 & 0,03 & 0,05 & 0,04 & 1,25 & 1,06 \\
\hline & 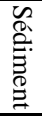 & 0,05 & 0,06 & 0,05 & 0,06 & 0,07 & 0,06 & 0,04 & 0,06 & 0,05 & 1,14 & 1,01 \\
\hline \multirow[b]{2}{*}{$\mathrm{Cr}$} & $\stackrel{\text { TI }}{\Xi}$ & 0,19 & 0,22 & 0,21 & 0,23 & 1,57 & 0,80 & 1,01 & 2,35 & 1,68 & 4,08 & 8,15 \\
\hline & 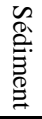 & 16,33 & 18,49 & 17,41 & 35,28 & 56,19 & 45,73 & 43,51 & 47,35 & 45,43 & 2,63 & 2,61 \\
\hline \multirow[b]{2}{*}{$\mathrm{Cu}$} & $\begin{array}{l}\text { T) } \\
\stackrel{\Xi}{\Xi}\end{array}$ & 1,86 & 2,23 & 2,05 & 3,67 & 5,98 & 4,83 & 2,76 & 4,61 & 3,69 & 2,36 & 1,80 \\
\hline & 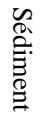 & 12,61 & 22,31 & 17,46 & 24,44 & 29,91 & 27,17 & 21,61 & 23,01 & 22,31 & 1,56 & 1,28 \\
\hline \multirow[b]{2}{*}{$\mathrm{Pb}$} & $\stackrel{\text { II) }}{\stackrel{\Xi}{\Xi}}$ & 3,09 & 4,13 & 3,61 & 0,16 & 18,86 & 9,51 & 10,68 & 20,13 & 15,40 & 2,63 & 4,27 \\
\hline & 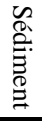 & 12,98 & 20,40 & 16,69 & 40,49 & 55,53 & 48,01 & 38,26 & 39,79 & 39,03 & 2,88 & 2,34 \\
\hline \multirow[b]{2}{*}{ Zn } & $\begin{array}{l}\text { T1 } \\
\stackrel{\Xi}{\Xi}\end{array}$ & 4,16 & 4,78 & 4,47 & 5,61 & 10,30 & 7,96 & 8,61 & 9,18 & 8,89 & 1,78 & 1,99 \\
\hline & 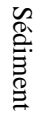 & 11,17 & 132,01 & 71,59 & 162,67 & 146,58 & 154,63 & 141,06 & 156,20 & 148,63 & 2,16 & 2,08 \\
\hline $\mathrm{Ni}$ & $\begin{array}{l}\text { T) } \\
\stackrel{\Xi}{\Xi}\end{array}$ & 3,23 & 4,01 & 3,62 & 5,01 & 6,06 & 5,54 & 7,01 & 5,72 & 6,37 & 1,53 & 1,76 \\
\hline
\end{tabular}




\begin{tabular}{|c|c|c|c|c|c|c|c|c|c|c|c|c|}
\hline & $\begin{array}{l}\text { 勇 } \\
\text { 总 }\end{array}$ & 17,23 & 17,87 & 17,55 & 19,61 & 18,01 & 18,81 & 17,45 & 17,87 & 17,66 & 1,07 & 1,01 \\
\hline \multirow[b]{2}{*}{$\mathrm{Al}$} & 永 & 0,10 & 0,31 & 0,21 & 0,32 & 0,57 & 0,45 & 2,01 & 2,66 & 2,34 & 2,18 & 11,39 \\
\hline & 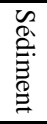 & 0,13 & 0,56 & 0,35 & 0,72 & 0,70 & 0,71 & 0,60 & 0,66 & 0,63 & 2,05 & 1,82 \\
\hline \multirow[b]{2}{*}{$\mathrm{Mn}$} & $\stackrel{\text { II) }}{\stackrel{\Xi}{\Xi}}$ & 1,51 & 2,02 & 1,77 & 2,49 & 2,71 & 2,60 & 1,63 & 1,94 & 1,78 & 1,47 & 1,01 \\
\hline & 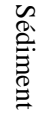 & 88,14 & 93,97 & 91,06 & 190,50 & 203,33 & 196,92 & 156,21 & 199,67 & 177,94 & 2,16 & 1,95 \\
\hline \multirow[b]{2}{*}{$\mathrm{Fe}$} & $\stackrel{\text { TI }}{\stackrel{\Xi}{\Xi}}$ & 27,81 & 123,72 & 75,77 & 346,23 & 265,32 & 305,78 & 196,97 & 295,89 & 246,43 & 4,04 & 3,25 \\
\hline & 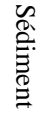 & 119,94 & 155,18 & 137,56 & 605,00 & 908,99 & 757,00 & 405,80 & 610,18 & 507,99 & 5,50 & 3,69 \\
\hline
\end{tabular}

Tableau IV : Teneurs des éléments métalliques dans trois stations R1, R2 et R3 de oued R'dom

\begin{tabular}{|c|c|c|c|c|c|c|c|c|c|c|c|c|}
\hline Variables & \multicolumn{4}{|c|}{ R1 } & \multicolumn{3}{|c|}{$\mathrm{R} 2$} & \multicolumn{3}{|c|}{ R3 } & \multirow[b]{2}{*}{ 离 } & \multirow[b]{2}{*}{$\widetilde{D_{\omega}}$} \\
\hline & & 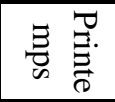 & $\stackrel{\vec{T}}{\vec{D}}$ & ప & 寻急 & $\begin{array}{l}\text { T. } \\
\vec{D}\end{array}$ & 官 & 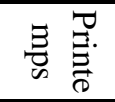 & $\stackrel{\mathbb{T}}{\vec{D}}$ & 灾 & & \\
\hline \multirow{2}{*}{$\mathrm{Cd}$} & Eau & 0,02 & 0,02 & 0,02 & 0,02 & 0,03 & 0,03 & 0,02 & 0,02 & 0,02 & 1,30 & 1,10 \\
\hline & Sédiment & 0,02 & 0,03 & 0,03 & 0,03 & 0,03 & 0,03 & 0,02 & 0,03 & 0,02 & 1,18 & 0,96 \\
\hline \multirow{2}{*}{$\mathrm{Cr}$} & Eau & 0,00 & 0,01 & 0,01 & 0,01 & 0,02 & 0,02 & 0,01 & 0,02 & 0,01 & 2,85 & 1,77 \\
\hline & Sédiment & 36,67 & 39,98 & 38,33 & 61,69 & 65,32 & 63,50 & 53,49 & 54,44 & 53,97 & 1,66 & 1,41 \\
\hline \multirow{2}{*}{$\mathrm{Cu}$} & Eau & 0,05 & 0,07 & 0,06 & 0,08 & 0,10 & 0,09 & 0,07 & 0,08 & 0,07 & 1,56 & 1,29 \\
\hline & Sédiment & 43,62 & 51,68 & 47,65 & 58,74 & 59,02 & 58,88 & 42,51 & 51,85 & 47,18 & 1,24 & 0,99 \\
\hline \multirow{2}{*}{$\mathrm{Pb}$} & Eau & 0,04 & 0,06 & 0,05 & 0,05 & 0,07 & 0,06 & 0,04 & 0,06 & 0,05 & 1,24 & 1,00 \\
\hline & Sédiment & 17,24 & 28,74 & 22,99 & 62,62 & 74,57 & 68,59 & 41,88 & 56,12 & 49,00 & 2,98 & 2,13 \\
\hline \multirow{2}{*}{$\mathrm{Zn}$} & Eau & 1,09 & 1,14 & 1,12 & 2,18 & 2,87 & 2,53 & 1,94 & 1,21 & 1,58 & 2,26 & 1,41 \\
\hline & Sédiment & 213,26 & 285,98 & 249,62 & 387,17 & 409,45 & 398,31 & 199,18 & 314,22 & 256,70 & 1,60 & 1,03 \\
\hline \multirow{2}{*}{$\mathrm{Ni}$} & Eau & 0,02 & 0,02 & 0,02 & 0,03 & 0,03 & 0,03 & 0,03 & 0,02 & 0,03 & 1,49 & 1,28 \\
\hline & Sédiment & 12,68 & 21,10 & 16,89 & 33,99 & 41,09 & 37,54 & 19,88 & 34,55 & 27,22 & 2,22 & 1,61 \\
\hline \multirow{2}{*}{$\mathrm{Al}$} & Eau & 0,07 & 0,21 & 0,14 & 0,68 & 0,99 & 0,84 & 0,23 & 0,88 & 0,55 & 5,97 & 3,93 \\
\hline & Sédiment & 1,04 & 3,90 & 2,47 & 1,89 & 4,21 & 3,05 & 1,63 & 3,96 & 2,80 & 1,24 & 1,13 \\
\hline \multirow{2}{*}{ Mn } & Eau & 0,14 & 0,16 & 0,15 & 0,14 & 0,18 & 0,16 & 0,14 & 0,16 & 0,15 & 1,09 & 0,99 \\
\hline & Sédiment & 102,00 & 257,70 & 179,85 & 281,20 & 296,80 & 196,95 & 134,08 & 215,24 & 174,66 & 1,10 & 0,97 \\
\hline \multirow{2}{*}{$\mathrm{Fe}$} & Eau & 2,90 & 3,35 & 3,12 & 3,66 & 7,55 & 5,60 & 2,55 & 5,01 & 3,78 & 1,80 & 1,21 \\
\hline & Sédiment & 119,98 & 196,67 & 158,33 & 262,71 & 329,79 & 296,25 & 216,78 & 252,24 & 234,51 & 1,87 & 1,48 \\
\hline
\end{tabular}

\section{Oued Beht}

Aux niveaux des stations étudiées, aussi bien pour l'eau que pour le sédiment, nombreux éléments métalliques ont présenté des valeurs 
minimales au printemps à B1et des valeurs maximales en été à B2. Nous en citons le $\mathrm{Cd}$, le $\mathrm{Cr}$, le $\mathrm{Cu}, \mathrm{l}$ 'Al et le $\mathrm{Mn}$; à ajouter également le Ni dans l'eau et le $\mathrm{Zn}$ et le Fer dans le sédiment. Dans les autres cas, toujours les teneurs minimales sont notées au printemps au niveau de la station B1 mais également les teneurs maximales sont notées au printemps de B2. Il s’agit de Zn et le Ni pour le sédiment.

En outre, dans chacune des stations étudiées, et pour tous les éléments métalliques étudiés, les valeurs notées en été sont plus élevées que celles de l'automne. Ainsi, aussi bien dans l'eau que dans le sédiment, le facteur «saison" est facteur déterminant des teneurs enregistrées des éléments métalliques que nous avons mesurés.

A noter également que l'abondance des éléments métalliques dans la station B2 s'explique par l'exposition directe de cette station aux rejets urbains et industriels déversées par la ville de Sidi Slimane. Contrairement, la station B1 a présenté des valeurs plus faibles car elle est située en amont de ces rejets. Ainsi, pour toute évaluation du degré métallique de toute station étudiée cette station (B1) peut être considérée comme la «station référence ».

Pour la station B3, les valeurs des teneurs métalliques sont relativement faibles par rapport à celles notées en B2. La situation B3 à 6 km en aval de B2 explique cette réduction des teneurs. En effet, comme ça été signalé par plusieurs auteurs, dans une eau courante, une fois qu'un élément métallique est déversé dans un cours d'eau, sa concentration se réduit progressivement le long du cours d'eau si sa teneur n'est enrichie ; une partie de la quantité initiale de cet éléments métallique sera fixée par le sédiment ou absorbée par les plantes ou les animaux du milieu ; et seule la partie qui reste sera drainée plus loin par le long du cours d’eau (Rodier, 2009).

\section{Oued R'dom}

Pour la distribution des teneurs des métaux étudiés le long des trois stations R1, R2 et R3, Celle-ci suit, à peu près, la même allure que celle notée pour la distribution de ces teneurs le long des stations B1, B2 et B3 de oued Beht. Ainsi, nous pouvons déduire pourquoi dans la station R1, qui se trouve en amont des rejets urbains de la ville (Sidi Kacem) et la moins polluée en éléments métallique, la station R2 qui est exposée directement aux rejets urbaines et industrielles est la plus polluée, et B3 qui est à 6 km en aval des rejets est moins polluée que la station $\mathrm{R} 2$.

\section{Teneurs stationnelles, orignes et dangers des éléments métalliques}

Cadmium, Cuivre, Plomb, Fer et Manganèse

Quoi que les valeurs notées ne varient beaucoup d'une station à l'autre, les valeurs moyennes les plus élevées de l'eau et du sédiment sont 
celles de B2 et R2. Les eaux des rejets urbaines et industrielles pourraient être donc une source de ces métaux. En effet, le cadmium est relâché dans l'environnement de façon naturelle notamment dans les rivières via l'usure de la roche traversée. Cependant, l’importante quantité du cadmium de l'environnement provient de l'activité humaine telle que la réduction du zinc, des engrais bio-industriels.

Dans la croute terrestre, le cadmium est présent toujours en combinaison avec du zinc (Airoldi et al., 1982 ; Ohkawara et al., 1996 ; Ohkawara et al., 1996). Mais, dans la nature, il peut aussi être issu de l'industrie comme sous-produit inévitable de l'extraction du zinc, du plomb et du cuivre et comme composant dans les pesticides et les engrais, Ainsi, il peut donc pénétrer dans l'environnement.

A travers la chaine alimentaire, des taux élevés de cadmium peuvent se développer dans le corps humains (Cassadou et Pouey, 2002). De même, l'Homme est exposé à l'absorption d'une certaine quantité de cadmium par voie pulmonaire près des sites de déchets dangereux ou des usines produisant ce métal. Puis, transporté jusqu'au foie par le sang, le cadmium se lie aux protéines et forme des complexes qui à leur tour sont transportés jusqu'aux reins endommagent ainsi leur fonctionnement.

Comme pour le chrome, le cuivre, élément métallique très fréquent dans le milieu naturel, peut être diffusé dans l'environnement par voie naturelle et par activité anthropique. En effet, il est très utilisé dans l'industrie et l'agriculture. Ainsi, le cuivre est présent dans beaucoup de type d'aliments, dans l'eau et dans l'air et le besoin du cuivre dans ces domaines ne cesse de s'accroitre. Notons qu'une exposition prolongée au cuivre peut provoquer une irritation à différentes parties de l'organisme et, pris à fortes dose, il peut bousier le rein et le foie et même entrainer la mort (RobertNadeau, 2012).

Le cuivre reste nécessaire en tant qu’élément essentiel pour la santé humaine. En effet, une carence en cuivre peut entrainer une anémie, des infections respiratoires fréquentes des troubles du sommeil, des problèmes de concentration et de mémorisation, des douleurs articulaires sans cause définie et des inflammations chroniques (Robert-Nadeau, 2012.).

Par ailleurs, quand le cuivre se trouve dans le sol ou le sédiment, il se lie fortement aux matières organiques et aux minéraux (Lenoir, 2006). Ainsi, il ne se déplace pas très loin et il ne pénètre presque jamais dans les eaux souterraines. Contrairement, dans les eaux de surface turbides, suspendue sur des particules de boue ou comme ion libre, le cuivre peut parcourir de longue distance. Ceci explique les différences des teneurs du cuivre entre B1 et B2 et entre R1 et R2.

Concernant le plomb $(\mathrm{Pb})$, c’est un métal présent à l'état naturel dans l'écorce terrestre. Mais, dans l'environnent il peut être issu de différentes 
activités industrielles. Pour l'eau potable, c'est lors de son passage dans des canalisations qui en contiennent que l'eau peut se charger en plomb. Ainsi, rejeté par voie naturelle, comme l'érosion du roc et du sol, ou relâché par les activités humaines, c'est lors de son passage dans des canalisations qui en contiennent que l'eau peut se charger en plomb; il peut se déposer sur les surfaces terrestres ou les plans d'eau et, par suite, s'accumuler dans le sol ou les sédiment (Pitrat et Braga, 2012).

Contrairement au cuivre, le plomb est un toxique dangereux pouvant avoir des conséquences sur la santé humaine (Garnier 2005 ; Pitrat et Braga, 2012). En effet, l' 'intoxication chronique par le plomb, même à faible dose peut agir sur les développements physique, intellectuel et psychomoteur de l'enfant. L'exposition au $\mathrm{Pb}$, même en petite quantité, peut être dangereuse pour les humains et la faune. Au Canada par exemple, le plomb est inscrit comme toxique (Loi canadienne sur la protection de l'environnement (1999)) (LCPE 1999).

Pour le fer $(\mathrm{Fe})$ et le manganèse $(\mathrm{Mn})$, ils sont souvent présents à l'état naturel ensembles et sont présents dans de nombreux types de roches (Donald Ellis, 1998). Les sources les plus fréquentes de fer et de manganèse dans l'eau souterraine sont naturelles en provenant par exemple de l'altération météorique des minéraux et des roches qui en contiennent (Donald Ellis, 1998). Ces deux éléments sont indispensables, en petites quantités, à tous les organismes vivants (Donald Ellis, 1998). D’autres sources artificielles sont sources du Fe et Mn notamment les effluents industriels, les eaux usées.

Par ailleurs, selon Denis Baize (1993), les teneurs en éléments traces métalliques peuvent être très variables au sein d'un même sol, d'un horizon à un autre, tout particulièrement dans le cas de sols à forte différenciation texturale, et ce en dehors de toute contamination anthropique (Segalen et Leal Silva, 1969). Les teneurs en éléments traces métalliques sont en effet fortement corrélées aux teneurs en argile et en fer (Chalmin, 2003).

Pour le manganèse, de récentes études ont noté que le manganèse dissout dans l'eau potable pourrait avoir un impact sur le développement neurologique de l'enfant, comme une baisse du quotient intellectuel (Zayed et al., 2003).

\section{Chrome, Zinc, Nickel et Aluminium}

Les teneurs moyennes du Cr, du Nickel et de l'Aluminium des stations exposées aux rejets urbains et industrielles (B2 et R2) et celles en aval de ces rejets (B3 et R3) sont plus élevées que celles notées en B1 et R1 qui sont en amont de ces rejets. Ceci peut être expliqué par l'existence de certaine quantité de ces métaux parmi les rejets urbains et industriels et la faible vitesse de son drainage le long du cours d'eau. A noter également que, 
sous l'influence de processus naturels et de l'activité humaine, plusieurs formes de chrome peuvent pénétrer dans l'air, l'eau et le sol. Ainsi, le chrome peut exister dans tous les compartiments de l'environnement.

Par ailleurs, certaines formes, tel que le chrome III, sont considérées comme non dangereuses et même utile à la santé humaine. Contrairement, à la forme VI est dangereuse (Ohkawara et al. 1996 ; Cassadou et Pouey, 2002). En effet, absorbé jusqu’à certain seuil, le chrome III est un nutriment essentiel pour l'homme et une carence de cette forme de chrome peut provoquer des problèmes au cœur, des perturbations du métabolisme et du diabète. Par contre la forme VI est dangereuse pour la santé, principalement pour les personnes travaillant dans l'industrie de l'acier et du textile, ou pour Les personnes qui fument du tabac (Dai et al., 2012 ; Tahri et al., 2014).

Par ailleurs, la plupart du chrome de l'air se dépose finalement et finit dans l'eau ou dans le sol mais le niveau de chrome dans l'air et dans l'eau est en général faible car arrivé au milieu aquatique ce métal est fixé sur les sédiments et devient immobile (Dai et al., 2012), seule une petite partie du chrome qui se retrouve dans l'eau finit par se dissoudre.

Concernant le zinc, il est présent naturellement dans l'air, l'eau et le sol et se trouver dans certains milieu en concentrations élevées sous l'effet des rejets issus des activités humaines. En effet, la plupart du zinc est rejeté par les activités industrielles, telles que l'exploitation minière la combustion du charbon et des déchets et l'industrie de l'acier. Ainsi, d'importantes quantités de zinc peuvent être trouvées dans le sol, puis par chaine alimentaire bio s'accumule et nuit à santé humaine (Pitrat et Braga, 2012). En outre, il est important de noter que sur un sol riche en zinc seul un nombre limité de plantes a des chances de survivre (Safa, 2015). Ainsi, une forte contamination par le zinc est une sérieuse menace pour la production des terres agricoles. Dans l'eau, la teneur du zinc peut atteindre des niveaux qui peuvent causer des problèmes de santé à cause des rejets industriels et des lieux de déchets toxiques (Klimek, 2012). Cependant, en petite quantité, le zinc est essentiel pour la santé de l'homme (Prasad, 1996 ; Wastney et al., 1996) .

Pour le nickel c'est un composé qui n’est présent dans l'environnement qu'à des concentrations très faibles (Cempel et Nikel, 2005). L'homme utilise ce métal pour différentes applications dont la plus commune est l'utilisation du nickel comme composants de l'acier ou d'autres produits métalliques. En outre, le nickel est relâché dans l'air par les centrales et les incinérateurs de déchets (Cempel et Nikel, 2005). L'homme peut être exposé au nickel en respirant, en buvant de l'eau, en consommant des aliments ou en fumant des cigarettes. En arrivant au sol, il se fixe sur ses éléments (Cempel et Nikel, 2005). Le nickel peut aussi finir dans les eaux de surfaces quand il est présent dans les eaux usées. En petites quantités, le nickel est essentiel 
mais, quand l'absorption est trop importante il peut présenter un risque pour la santé humaine et animale (Mandal et al., 2002).

Concernant l'aluminium, dans le milieu naturel il se forme au cours de l'érosion des feldspaths; de même, un certain nombre de pierres précieuses contiennent de l'aluminium, comme par exemple les rubis et les saphires. En outre, l'aluminium est présent dans les sols sous la forme d'hydroxide, insoluble dans l'eau (Godfrin et Van Bladel, 1990 ; Cherifi, 2013). . La dissolution des ions Al3+ est toxique pour les plantes car cette opération affecte les racines et diminue la consommation de phosphate (Boyer, 1976). En outre, lorsque la valeur du pH est sous 4,5, la solubilité de l'aluminium augmente rapidement (Godfrin et Van Bladel, 1990 ; Cherifi, 2013) d'où une augmentation de la concentration en aluminium au-delà de 5 ppm. Cette réaction peut également se produire lorsque les valeurs du $\mathrm{pH}$ sont très hautes. A signaler que, quoi que son rôle est essentiel pour la vie, l'aluminium peut affecter de manière négative la vie terrestre et aquatique. La prise de grande quantité d'aluminium peut avoir des effets néfastes sur la santé humaine, notamment chez les gens souffrant de problème de reins. L'aluminium. Il endommage les nerfs et augmente les risques d'allergie. On suspecte également une corrélation entre la consommation d'aluminium et la maladie d'Alzheimer (Gourier-Fréry et al., 2004).

\section{Bilan de la contamination métallique des eaux : Indice de contamination}

Pour un élément métallique donné, l’évaluation de la teneur n’est pas suffisante pour évaluer le degré contamination du milieu par cette élément. Ainsi, cette première évaluation de contamination du milieu peut être complétée le calcul d'un l'indice de contamination métallique (Ic).

Rappelons que ce sont les stations B1 et R1 qui ont été prises comme stations de référence respectives aux deux cours d'eau étudiés. Les valeurs des indices de contamination des eaux de Oued R'dom et oued Beht sont supérieure à 1. Autrement dit toutes les stations présentent un degré de contamination métallique supérieur à celui de la station référence (B1 pour Oued Beht et supérieur à celui de R1 pour Oued R'dom).

Pour les deux cours d'eau étudiés, L’évolution spatiale de l'Ic moyen de l'eau révèle la présence d'une contamination élevée en B2 pour Oued Beht et en R2 pour Oued R'dome. En effet, les valeurs de contaminations moyennes respectives aux deux cours d'eau sont 1,52 pour B2 et 1,43 pour B3 ; alors que ces valeurs sont que de 42,46 pour R2 et 52,47 pour B3. A rappeler que ce sont les stations B2 et R2 qui sont les plus exposées aux rejets urbains et industrielles de leurs villes riveraines ce qui explique leur degré important de pollution métallique. Au contraire, les stations situées à 6 km en aval de ces rejets c'est-à-dire B3 et R3 ont présenté Ic de valeurs plus faibles mais les teneurs des éléments métalliques restent comme même plus 
élevées que celles notées aux niveaux de B1 et R1. Ceci s’explique par le phénomène d'autoépuration des cours d'eau étudiés et de la fixation par le sédiment, les plantes et les animaux de certaines quantités des éléments métalliques déversées aux niveau de B2 ou R2.

Par ailleurs, dans l'eau et dans le sédiment, de B2, presque pour tous les éléments métalliques les valeurs de Ic sont inférieures à 3. Ainsi, selon la grille de classification relative aux valeurs de Ic, le degré de contamination de la station B2 par ces éléments dont l'Ic est inférieur à 3 est faible. Nous en citons le cadmium et le nickel, Contrairement pour le chrome dans l'eau et le fer dans l'eau et dans le sédiment, les valeurs sont supérieures à 4 est inférieures à 10. Par suite le degré de contamination du milieu par ces éléments est moyen.

Concernant la station B3, pour presque tous les éléments métalliques les Ic évalués sont inférieurs à 3. La décontamination de B3 par ces éléments métalliques est donc faible. Trois Ic font exception par valeur supérieur à 4 dans l'eau, le plomb (Ic = 4,265), le chrome $(\mathrm{Ic}=8,153)$ et le fer $($ Ic= 11,393). Ainsi, dans de la station B3, la contamination de B3 par le plomb chrome est moyenne, et est importante par le fer.

Concernant la comparaison des Ic notés en B2 (IcB2) et ceux de B3 (IcB3), nous pouvons diviser les valeurs en deux catégories :

Une première catégorie ayant un IcB2 $\geq \mathrm{IcB} 3$. Pour cette catégorie d'éléments métalliques, une certaine quantité de la totalité présente en B2 de l'élément métallique s’est fixé sur le sédiment, s’est assimilée par les plantes ou les animaux du milieu ou a été drainée au de-là de B3. Nous en citons comme exemple le Manganèse.

Une deuxième catégorie d'élément métallique dont l'Ic de B3 est $\geq$ à l'Ic de B2. Pour cette catégorie d'éléments métalliques la tranche du cours d'eau étudiée a des difficultés à réduire la quantité du métal existant au niveau de B2 au fur et à mesure que la quantité notée en B2 se déplace vers B3; Ainsi, une accumulation de certains éléments métalliques a été effectuée.

Concernant les Ic notés aux niveaux de R2 et R3 de Oued R'dom, l'aluminium dans l'eau est le seul métal ayant un Ic supérieur à 4 et égale à 5,968 en R2. Par suite, le degré de contamination de R2 par ce métal est considéré comme appartenant à la classe moyenne. Pour les autres Ic toutes les valeurs calculées sont inférieurs à 4 ; il s’agit donc de métaux ayant des degrés de contaminations moyennes dans l'eau ou dans le sédiment. En outre, pour la totalité des éléments évalués, les valeurs de Ic notées au niveau de R3 (IcR3 ; tab. IV) sont inférieures à celles de Ic de R2 (IcR2 ; tab. III). Il semble donc que pour les deux tranches des deux cours d'eau prospectés, Oued R'dom a plus d'aptitude de réduire son degré de contamination métallique que Oued Beht. A noter que comme a été signalé par Oraison et 
al. (2010), cette aptitude dépend de la topographie du cours d’eau, de la richesse qualitative et quantitative de sa biodiversité et de l'importance de son débit.

\section{Conclusion}

Pour la plupart des paramètres physico-chimiques et des éléments métalliques, les valeurs ou les teneurs les plus élevées sont notées en été. La réduction du débit des cours d'eau et l'importance de l'évaporation pendant cette saison entrainent une concentration varieée de plusieurs éléments chimiques du milieu. Parmi ces éléments nous citons les chlorures, la dureté total, calcique ou magnésique, les orthophosphates, les sulfates. A la suite de la concentration de ces éléments des facteurs physiques peuvent changer de valeur tels le $\mathrm{pH}$ et la conductivité. Egalement, le taux d'oxygène dissous de l'eau noté au printemps est plus élevé en automne qu'en été ; la diminution de l'abondance des végétaux du milieu aquatiques explique cette différence. Ainsi, pour toutes les valeurs ou les teneurs des variables physico-chimiques étudiés, nous pouvons conclure que le facteurs «Saison » a un effet sur les caractéristiques physico-chimiques et métalliques du milieu prospecté.

Par ailleurs, la comparaison des valeurs de plusieurs paramètres physico-chimiques et métalliques nous a montré que ce sont les zones les plus exposées aux rejets des eaux urbaines et industrielles des deux villes qui sont les plus polluées par des substances organiques, des éléments métalliques et des substances non biodégradables et que cette pollution est plus accentuée en été. De même, les faibles valeurs des paramètres physicochimiques et teneurs des éléments métalliques des zones des cours d'eaux qui se trouvent à $6 \mathrm{~km}$ en aval des zones directement exposées aux rejets montrent que Oued R'dom et Oued Beht possède un pouvoir d'autoépuration de la grande partie des substances polluantes et que ce pouvoir est légèrement plus élevé chez Oued R’dom.

\section{References:}

Abdellaoui A., 1990. Contribution à l'étude de la pollution des cours d'eau marocains par les métaux lourds -cas de oued Boufekrane : Thèse de 3éme cycle, Université Moulay Ismail, Meknès, 258p.

Abouzaid et Duchesne. (1984).Direction contrôle qualité des eaux. ONEP. Bulletin officiel (2002) Grilles de qualité des eaux potables, 10 Chaabane 1423 (17 octobre 2002). Ministère de l'aménagement du territoire, de l'urbanisme, de l'habitat et de l'environnement, Rabat.

Alonso A., 2004. Les peuplements de poissons comme outil pour la gestion de la qualité environnementale du réseau hydrographique de la Garonne. Thèse présentée pour obtenir le titre de Docteur de l'Institut National Polytechnique de Toulouse Ecole Doctorale de Sciences Ecologiques, 
Vétérinaires, Agronomiques et Bio-ingénieries Spécialité en Sciences Agronomiques. 134 pp.

Alenka L., David C., 1996. Hydrogen-bond Kinetics in Liquid Water. Nature 1996, 379: 55. 10.1038/379055a0

Anonyme, 2010 ; État de l'environnement, Références juin 2010 [archive], L'environnement en France, Commissariat général au développement durable - Service de l'observation et des statistiques (p. 41-42 / 152)

Azzaoui S. 1999. Les métaux lourds dans le bassin versant du Sebou : Géochimie, sources de pollution et impact sur la quantité des eaux de surface : Thèse de doctorat national. Univ Ibn tofail : Kenitra, Maroc : 138 p.

Badraoui M., Agbani M. \& Soudi B., 2000. Qualité des sols sous mise en valeur intensive Séminaire 'Intensification agricole et qualité des sols et des eaux’, Rabat, 2-3 Novembre 2000. Evolution de la qualité des sols sous mise en valeur intensive au Maroc ; Institut Agronomique et Vétérinaire Hassan II, Rabat , Maroc

Baraud F., Tellier S., Astruc M., 1999, Temperature effect on ionic transport during soil electrokinetic treatment at constant $\mathrm{pH}$. Journal of hazardous materials 64: 263-281.

Berdai H., B. Soudi, M. Badraoui et A. Bellouti, 2004. Détermination de l'ammonium non échangeable des sols du Tadla (Maroc) : Potentiel et Evolution ; Revue H.T.E. N 128 - Mars 2004

Bertolini D, Tani A., 1995. Stress Tensor and Viscosity of Water: Molecular Dynamics and Generalized Hydrodynamics Results.Phys Rev E 1995, 52: 1699. 10.1103/PhysRevE.52.1699

Blieffert C. et Perraud R. 2001. "Chimie de l'environnement - air, eau, sols, déchets», ed. DeBoeck Université. ?

Boutayeb M., Bouzidi A. \& Fekhaoui M., 2012. Etude de la qualité physico-chimique des eaux usées brutes de cinq villes de la région de la Chaouia-Ouardigha (Maroc). Bulletin de l'Institut Scientifique, Rabat, section Sciences de la Vie, 2012, nº 34 (2), p. 145-150.

Boyer J. 1976. L'aluminium échangeable : incidences agronomiques, évaluation et correction de sa toxicité dans les sols tropicaux. Cah. ORSTOM, sér. Pédol., vol. XIV, no 4, 1976 : 259-269.

Cassadou S., 2002. Evaluation des risques sanitaires lies a la présence de cadmium dans la rivière Lot, entre Boisse-Penchot et Aiguillon. Étude réalisée à la demande de la DRIRE Midi-Pyrénées et cofinancée par la DRIRE Midi-Pyrénées et l'Agence de l'Eau Adour Garonne. Rapport final Septembre 2002

Cempel M., Nikel G., 2005. Nickel, 2006. A Review of Its Sources and Environmental Toxicology. Polish J. of Environ. Stud. Vol. 15, No. 3, 375382 
Chahboun M., Abdelkader C. et Abdelhamid Z., 2014. Étude de la qualité des eaux d'une retenue située sous climat aride : cas du barrage Hassan II (Province de Midelt, Maroc). Afrique SCIENCE 10(2) (2014) 199 - 212199 ISSN 1813-548X, http://www.afriquescience.

Chartrand, J., Levallois, P., Gauvin, D., Gingras, S., Rouffignat, J. et Gagnon, M.-F., 1999. La contamination de l'eau souterraine par les nitrates à l'île d'Orléans, Vecteur Environnement, 32(1), 37-46.

Cherifi M., 2013. Décontamination électrocinétique d'une boue d'eau potable contenant de l'aluminium. Thèse Présentée en vue de l'obtention du diplôme de Doctorat en Science Spécialité Chimie analytique ; Université Mokhtar Badji (111 P.)

Chikou A. 2006. Etude de la démographie et de l'exploitation halieutique de six espèces de poissons-chats (Teleostei, Siluriformes) dans le delta de l'Ouémé au Bénin. Thèse de Doctorat, Université de Liège, Liège, p. 459.

Claudio A., Aecio P., and Francisco P., 1982. Thermochemical study of adducts of methylurea with zinc, cadmium, and mercury halides. CAN. J. CHEM. VOL. 60, 1982, pp: 2132-2136

Deby C. et Deby-Dupont G., 1993. L'oxygène et la vie: tome 1 - Initiation au métabolisme de l'oxygène ; Chapitre XII: Nécessité d'O2 pour la vie aérobie: Production d'énergie ; Université de Liège - Centre de l'Oxygène, Recherche et Développement (CORD)

Denis B., 1993. Teneurs totales en métaux lourds dans les sols français premiers résultats du programme. Courrier de l'Environnement de l'INRA n²2 37 ASPITET par INRA, unité de Science du sol, 45160 Ardon

Donald O., 1998. Enlèvement du fer et du mangan7se de l'eau souterraine par oxydation et par microfiltration. Mémoire prés enté à la Faculté des études supérieures de l'université Laval pour l'obtention du grade de Maître ès sciences (M. Sc.) Département de génie civil Faculté des Sciences et génie Université Laval, 1998

Droge W., 2002. Free radicals in the physiological control of cell function. Pour le rôle des ROS dans la transmission des signaux intra-cellulaires Physiological Review. 2002; 82: 47-95.

El Blidi S., Fekhaoui M., 2003. Hydrologie et dynamique marégraphique de l'estuaire du Sebou (Gharb, Maroc). Bull. Inst. Sci., 25, 57-65.

Elhassan V., Belghyti D., Elibaoui H., Benabid M., 2012. Ecological status of water ecosystem where live common trout in the central middle morocco: Sidi Rachid river. Ecological status of Sidi Rachid river Journal of Science Research N 4, Vol. 1, p. 40-45.

Emilie C., 2003. Caractérisation des oxydes de manganèse et usage des pigments noirs au paleolithique supérieur. Thèse de l'Université de MarneLa-Vallée Institut Francilien; présentée et soutenue publiquement le 14 novembre 2003 
Ezzaouak M., 1991. La caractérisation hydrodynamique physico-chimique et bactériologique des eaux superficielles de l'estuaire de Bouregreg (Maroc) soumis aux rejets des villes de Rabat et Salé. Thèse Doct. 3ème cycle. Univ. Mohammed V (Rabat), $140 \mathrm{p}$

Fekhaoui A., Bennasser L., Bouachrine M., 1996. Utilisation d'un nouvel indice d'évaluation de la contamination métallique des sediments: cas du bas Sebou (Maroc). Bllll, lnsl. Sei., Rabat, 1996, N²0, p. 143-150

Garnier R., 2005. Toxicité du plomb et de ses dérivés (Toxicity of lead and lead compounds). EMC - Toxicologie-Pathologie, Volume 2, Issue 2, June 2005, Pages 67-88

Godfrin J.-M. et Van Bladel R., 1990. Influence du pH sur l'adsorption du cuivre et du zinc par les sols. Science du Sol - 1990 - Vol. 28, 1, pp. 15-26

Gourier-Fréry C., Fréry N., Claudine B., Sylvaine C., Robert G., Hubert I., Coralie R., Claude R., 2004. Aluminium Quels risques pour la santé ? Synthèse des études épidémiologiques Volet épidémiologique de l'expertise collective InVS-Afssa-Afssaps. Rapport constitue le volet épidémiologique détaillé de l'expertise collective InVS-Afssa-Afssaps « Evaluation des risques sanitaires liés à l'exposition de la population française à l’aluminium ».

Gulis G., Czompolyova M., Cerhanw J. R., 2001, An Ecologic Study of Nitrate in Municipal Drinking Water and Cancer: Incidence in Trnava District, Slovakia. Environ. Res. Section A, 88, 182-187.

Himmi N., Fekhaoui M., Foutlane A., Bourchich H., El Maroufy M., Benazzou T. \& Hasnaoui M. 2003. Relation planctondescripteurs physique et chimique dans un bassin de maturation (Lagunage Mixte Ben Slimane Maroc).. Riv. Idrobiol., Perugia, 42, 1-3, 107-129.

Jun Dai, FengLian Ren, and ChunYuan Tao, 2012. Article Adsorption of $\mathrm{Cr}(\mathrm{VI})$ and Speciation of $\mathrm{Cr}(\mathrm{VI})$ and $\mathrm{Cr}(\mathrm{III})$ in Aqueous Solutions Using Chemically Modified Chitosan. Int. J. Environ. Res. Public Health 2012, 9, 1757-1770

Kestemont, P., Goffaux, D. Breine, J., Belpaire, C., de Vocht, A., Philippart, J.C., Baras, E., Roset, N., De Leeuw, J. \& Gérard, P., 2002. Fishes of the River Meuse: biodiversity, habitat influences and ecological indicators, In ICM, Proceedings of the First International Scientific Symposium on the River Meuse. November 27-28, 2002, Maastricht, 84-90

Klimek B., 2012. Effect of Long-Term Zinc Pollution on Soil Microbial Community Resistance to Repeated Contamination. Bull. Environ. Contam. Toxicol. 2012 Apr; 88(4): 617-622.

Mercoiret L., 2010. Conception et exploitation des stations d'épuration des petites et moyennes collectivités » Qualité des eaux usées domestiques produites par les petites collectivités Application aux agglomérations 
d’assainissement inférieures à 2000 Equivalent Habitants, Rapport final Léa Mercoiret Cemagref Novembre 2010

Leclercq L., 2001. Les eaux courantes : caractéristiques et moyens d'étude, dans Les zones humides. Actes des colloques organisés en 1996 par le Ministère de la Région Wallonne dans le cadre de l'Année Mondiale des Zones Humides, Jambes, Région Wallonne, DGRNE. pp. 67-82.

Lehninger AL., Nelson DL., Cox MM., 1993. Principles of Biochemistry; Pour la respiration mitochondriale : Worth Publ. ed., 1993, pp. 542-597.

Lepengue AN., Mouaragadja I., M’batchi B., 1997. Effet du chlorure de sodium ( $\mathrm{NaCl}$ ) sur la germination et la croissance du maïs (Zea mays L., Poaceae) au Gabon. International Journal of Biological and Chemical Sciences. Vol 4, No 5 (2010) ISSN: 1991-8631 (print), ISSN: 1997-342X (online).

Levallois, P. et Phaneuf, D. (1994), La contamination de l'eau potable par les nitrates : analyse des risques à la santé, Revue canadienne de santé publique, 85(3), 192-196.

Loez C. R., A. Saliban et M. L. Topalian, 1998. Associations phytoplanctoniques indicatrices de la pollution par le zinc. Revue des sciences de l'eau / Journal of Water Science, vol. 11, n 3, 1998, p. 315-332.

Loi canadienne sur la protection de l'environnement (1999) (LCPE 1999)). Guide expliquant les caractéristiques principales de la Loi canadienne sur la protection de l'environnement (1999) [LCPE]

Mandal R., Hassan NM., Murimboh J., Chakrabarti CL., Back MH., Rahayu U., Lean DR., 2002. Chemical speciation and toxicity of nickel species in natural waters from the Sudbury area (Canada). Environ Sci Technol. 2002 Apr 1;36(7):1477-84.

Mittal CK, Murad F., 1979. Activation of guanylate cyclase by superoxide dismutase and hydroxyl radical: a physiological regulator of guanosine 3',5'monophosphate formation; Pour le rôle des ROS dans la transmission des signaux intra-cellulaires. Proceedings of the National Academy of Sciences U S A. 1977; 74: 4360-4.

Nordine N., Driss K., Mohamed H., 2015. Assessment of physico-chemical quality of groundwaterof the Jurassic aquifers inhigh basin of Ziz (Central High Atlas, Morocco). J. Mater. Environ. Sci. 6 (4) (2015) 1068-1081 Nouayti et al. ISSN : 2028-2508 CODEN: JMESCN 1068

Ohkawara S., Kaji T., Yamamoto C., Fujiwara Y., Sakamoto M, Kozuka H., 1996. Interaction between cadmium and zinc in the production and sulfation of glycosaminoglycans in cultured bovine vascular endothelial cells. J Toxicol Environ Health. 1996 Feb 9;47(2):183-93.

Oraison F., Yves S., Kris Van Looy. Partenariat 2010, Restauration des milieux aquatiques Restaurer l'hydromorphologie des cours d'eau et mieux maîtriser les nutriments : une voie commune ? Synthèse bibliographique 
Version finale. Pôle hydroécologie des cours d'eau Onema-Cemagref Lyon Mars 2011

Pitrat J. et Braga B., 2012. Évaluation de la contamination métallique d’une ressource en eau de la ville de Curitiba, Brésil. VERTIGO, revue électronique en sciences de l'environnement. Volume 12 Numéro 1 | mai 2012

Prasad AS., 1996. Zinc deficiency in women, infants and children. Am Coll Nutr 1996, 15: 113-120

Ribolzi O., V. Valles, L. Barbiero, 1993 ; avec la collaboration technique de S. SES et M. Monjusiau, 1993. Contrôle géochimique des eaux par la formation de calcite en milieu méditerranéen et en milieu tropical. Arguments d'équilibre et argument de bilan. Science du Sol - 1993 - Vol. 31, 1/2,77-95

Robert-Nadeau F., 2012. Évaluation des risques toxicologiques et écotoxicologiques d’un terrain contaminé par des métaux. Essai présenté au Centre universitaire de formation en environnement en vue de l'obtention du grade de maitre en environnement. Centre Universitaire de Formation en environnement Université de Sherbrooke, Sherbrooke, Québec, septembre 2012

Rodier J., 1984. L’analyse de l'eau : Eaux naturelles, eaux résiduaires, eaux de mer. Edition Dunod Paris.

Rodier J. (2009). L'analyse de l'eau - eaux naturelles, eaux résiduaires, eau de mer, 9ème édition, Paris, Dunod, 1475 p

Messeaouda S., 2015. Etude de la capacité de rétention et d’élimination des cations métalliques par des adsorbants naturels. Thèse de Docteur en Sciences Spécialité : Sciences de la Nature et de la Vie; Université de Mustapha Stambouli, Mascara Faculté des Sciences de la Nature et de la Vie l’université de Mascara. 184 pp.

Santé Canada, 1992; Aliments et nutrition. Les guides alimentaires canadiens, de 1942 à 1992

Segalen P. et J. N. Leal Silva, 1969. Le fer, le manganèse, l'aluminium et la silice facilement extractibles dans les sols tropicaux a sesquioxydes. Cah. O.R.S.T.O.M., sér. Pédol.. vol. VII. no 3. 1969.

Soaresa M., Vale M., Vasconcelos V.., 2011. Effects of nitrate reduction on the eutrophication of an urban man-made lake (Palácio de Cristal, Porto, Portugal). Environ Technol. 2011 Jul;32(9-10):1009-15.

Tahri N., Sayel H., Bahafid W. \& El Ghachtouli N., 2014. Effet des polyamines sur la réduction du chrome hexavalent par des souches bactériennes et leur résistance. Biotechnologie, Agronomie, Société et Environnement? P: 1780-4507, Volume 18 (2014) numéro 4

Lenoir T., 2006. Mécanismes de rétention du cuivre dans les sols : évaluation statistique des approches macroscopiques et spectroscopiques. 
Thèse pour obtenir le grade de Docteur de l’Université de Grenoble et soutenue publiquement le « 3 octobre 2011 »

Walmsley J., 2002. Editorial. British Journal of Learning Disabilities, 30: 12. doi: 10.1046/j.1354-4187.2002.00176.x

Wastney Me., Angelus P., Barnes Rm., Siva Subramanian Kn, 1996. Zinc kinetics in preterm infants: a compartmental mode1 based on stable isotope date. Am] Physiol1996, 271 : R1452-R1459

Zayed J., Panisset M., Mergler D., 2003. Système nerveux, in Environnement et Santé publique - Fondements pratiques. Manuel publié en 2003 (Chapitre 27) pp. : 699-712.

Zhao XF, Yang LR, Shi Q, Ma Y, Zhang YY, Chen LD, Zheng HF., 2008. [Nitrate pollution in groundwater for drinking and its affecting factors in Hailun, northeast China]. Huan Jing Ke Xue. 2008 Nov; 29 (11) : 2993-8.

Zhou Yue, 2010. Evaluation de la biodisponibilité des métaux dans les sédiments Juin 2009 Étude réalisée ; Etude réalisée dans le cadre de la convention de coopération n ${ }^{\circ} 64556$ entre l'Agence de l'Eau Artois-Picardie et l'Université des Sciences et Technologies de Lille I pour la période 2008 2010. 